\title{
Stochastic Analysis of a Computer System with Hardware Redundancy and Failure of Service Facility during Repair
}

\author{
Amit Kumar \\ Department of Statistics \\ M.D. University \\ Rohtak-124001, Haryana, India
}

\author{
S. C. Malik \\ Department of Statistics \\ M.D. University \\ Rohtak-124001, Haryana, India
}

\begin{abstract}
A stochastic model for a computer system is developed by providing hardware redundancy in cold standby. The hardware and software are considered as the two main components of the system having operative and complete failure modes. A single service facility is used to repair the hardware component and also to upgrade the software. The service facility is subjected to failure while repairing the hardware. Treatment is given to the failed service facility so that it can resume the assigned repair activities with full efficiency. The up-gradation of the software is done either at its outdated features or when it fails to follow the instructions properly in order to complete the assigned jobs. The failure times of the components and service facility follow negative exponential distribution while the distributions of hardware repair, software up-gradation and treatment time of the service facility are taken as arbitrary with different probability functions. The expressions for some reliability measures are derived in steady state by using semi-Markov process and regenerative point techniques. The graphical study of these measures has also been made for arbitrary values of the parameters.
\end{abstract}

\section{Keywords}

Computer System, Hardware Redundancy, Failure of Service Facility, Reliability Measures and semi-Markov Process

\section{INTRODUCTION}

In the fast growing age of digitalization the significance of the usages of computer systems with internet connection has increased many folds in our life. We are creating new kinds of habits such as listing music on the computer, online money earning, online money transaction, running an online business, taking online classes and online booking of transportation facilities, etc. Also, we are getting a solution with high speed and accuracy because of the use of computers in official works. And, Microsoft office package, email, video conferencing tools, etc. are the few vital applications in this direction that speed the official work with accuracy. As a result this leads to the need to specify and design computing systems which could fulfill the requirements of targeted applications at the list possible expenditure. There has been a challenge to the system designers and reliability engineers to develop a system to perform its intended job at least for a specific duration. The scholars of the field have suggested several techniques for enhancing life span and performance of computer systems. The technique of cold standby redundancy has been adopted in order to provide services to the costumers for a considerable period. Over the years, some research papers on the reliability improvement techniques of computer systems have been written by applying unit wise and component wise redundancy methods. Malik and Anand $(2010,12)$ and Kumar et al. (2013) analyzed the reliability characteristics of computer systems with unit wise redundancy in cold standby. The researches indicate that component wise redundancy is better than that of unit wise redundancy so far as reliability is concerned. Malik and Munday (2014) described a stochastic model for a computer system by providing hardware redundancy in cold standby. In most of these studies the system reliability models have been developed under a common assumption that service facility neither fails nor deteriorates. This assumption seems to be unrealistic in case service facility (online/offline) meets with an accident may because of mishandling of the system, lack of knowledge about the functioning of the system, poor judgment skills of the server and many more. In such situations treatment may be given to the service facility in order to resume the assigned jobs. Malik and Dhankar (2010) have used the idea of server failure while analyzing reliability characteristics of a single unit system. Nandal and Rathee (2015) made a stochastic analysis of a redundant system with server failure.

The main aim of this paper is to analyze some reliability characteristics of a computer system with component wise redundancy and failure of service facility. A stochastic model for a computer system is developed by providing hardware redundancy in cold standby. The hardware and software are considered as the two main components of the system having operative and complete failure modes. A single service facility is used to repair the hardware component and also to upgrade the software. The service facility is subjected to failure while repairing the hardware. Treatment is given to the failed service facility so that it can resume the assigned repair activities with full efficiency. The up-gradation of the software is done either at its outdated features or when it fails to follow the instructions properly in order to complete the assigned jobs. The failure times of the components and service facility follow negative exponential distribution while the distributions of hardware repair, software up-gradation and treatment time of the service facility are taken as arbitrary with different probability functions. The expressions for some reliability measures such as mean time to system failure (MTSF), availability, busy period of the server due to hardware repair and software up gradation, expected number of software up gradation, expected number of treatments given to the server and finally the profit function are derived in steady state by using semi-Markov process and regenerative point techniques. The graphical study of these measures with respect to failure rate of the service facility (server) has also been made for fixed values of other parameters. 


\section{State Transition Diagram}

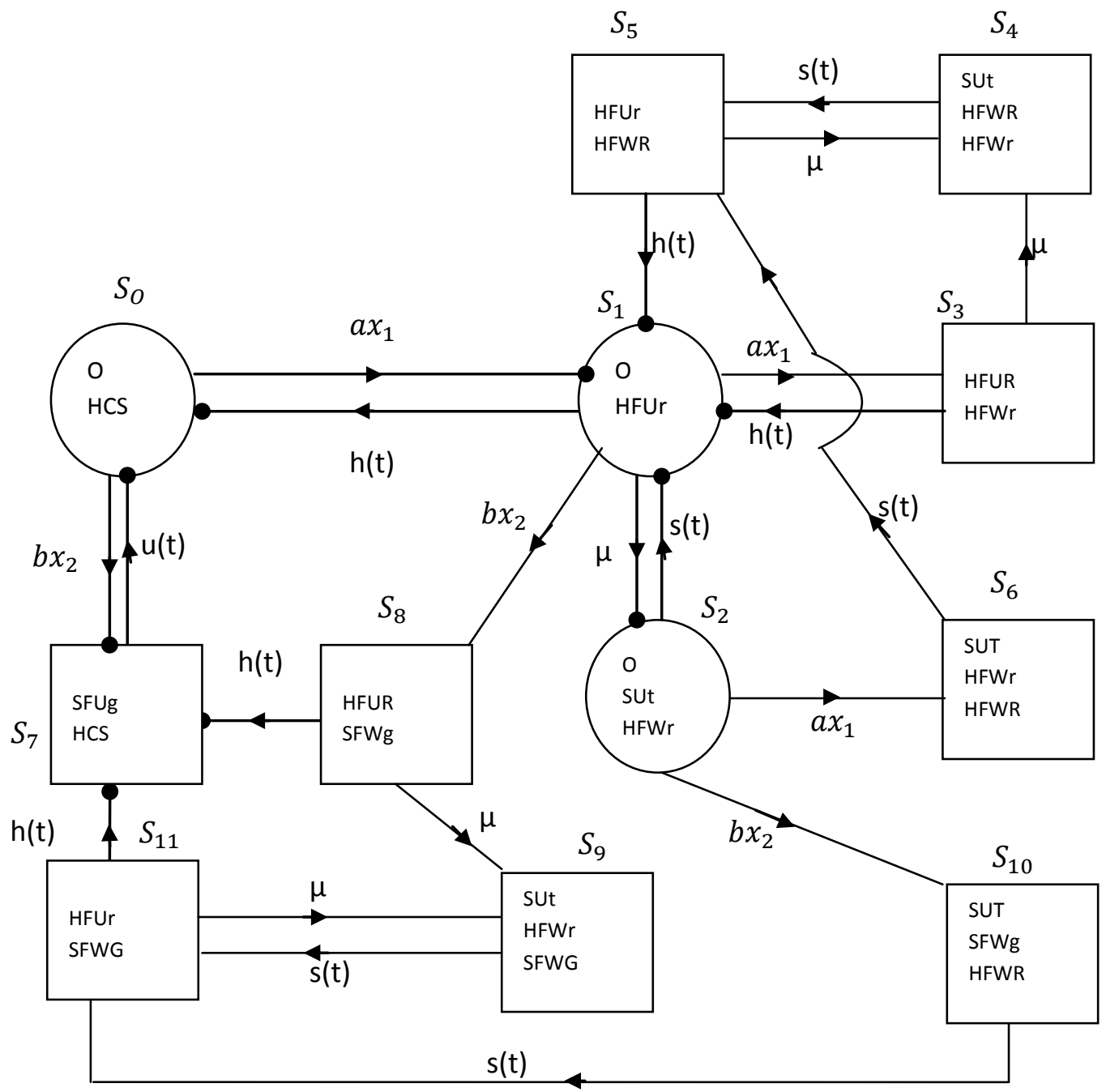

Fig.: 1

\section{Operative State}

\section{NOTATIONS:}

E : The set of regenerative states

$\mathrm{O} \quad$ : The unit is operative and in normal mode

HCS : The unit hardware cold-standby

$x_{1} / x_{2} \quad: \quad$ Constant hardware/ software failure rate

HFUr/HFUR : $\quad$ The unit is failed due to hardware and is under repair / under repair continuously from previous state

HFWr/HFWR : The unit is failed due to hardware and is waiting for repair / under repair continuously from previous state

SFUg/SFUG : The unit is failed due to software and is under replacement / under replacement continuously from previous state

\section{: Regenerative Point}

SFWg/SFWG : The unit is failed due to the software and is waiting for replacement/ waiting for replacement continuously from previous state

$\mathrm{h}(\mathrm{t}) / \mathrm{H}(\mathrm{t}) \quad$ : $\quad$ pdf/cdf of repair time of the unit due to hardware failure

$\mathrm{u}(\mathrm{t}) / \mathrm{U}(\mathrm{t}) \quad$ : $\quad \mathrm{pdf} / \mathrm{cdf}$ of replacement time of the software

SUt/SUT : The unit is failed due to the software and under treatment/ under continuously treatment

$\mathrm{s}(\mathrm{t}) / \mathrm{S}(\mathrm{t})$

$\mu$

$q_{i j . k}(t)$

$/ Q_{i j . k}(t)$ $\mathrm{pdf} / \mathrm{cdf}$ of the server under treatment server failure rate regenerative state $S_{i}$ to a regenerative state $S_{j}$ or to a failed state $\mathrm{pdf} / \mathrm{cdf}$ of direct transition time from 
$S_{j}$ visiting state $S_{k}$ once in $(0, \mathrm{t}]$

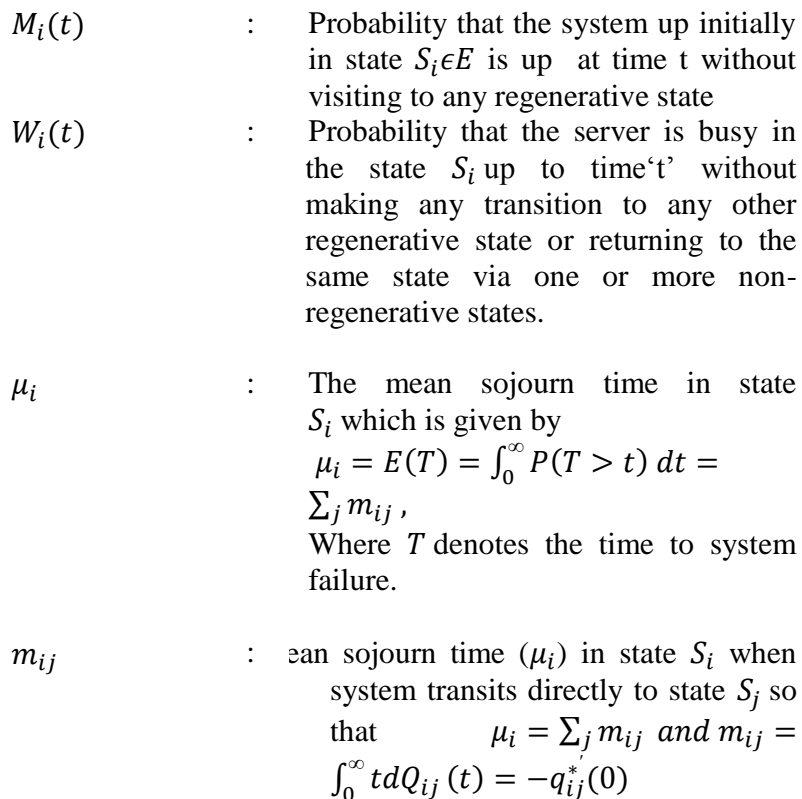

\&/C : Symbol for Laplace-Stieltjes convolution/Laplace convolution */** : Symbol for Laplace Transformation (LT)/Laplace Stieltjes Transformation (LST)

The following are possible transition states of the system:

S0 = (O, HCS $), \mathrm{S} 1=(\mathrm{O}, \mathrm{HFUR}), \mathrm{S} 2=(\mathrm{O}, \mathrm{SUt}, \mathrm{HFWr}), \mathrm{S} 3=$ (HFUR,HFWr)

S4 = (SUt,HFWR,HFWr), S5 = (HFWr,HFWR $),$ S6 = (SUT,HFWr,HFWR)

$\mathrm{S} 7=(\mathrm{SFUg}, \mathrm{HCS}), \quad \mathrm{S} 8=(\mathrm{HFUR}, \mathrm{SFWg}), \quad \mathrm{S} 9=$ (SUt,HFWr,SFWG)

S10 $=($ SUT, SFWg, HFWR $)$, S11 = (HFUr, SFWG $)$

The states S0, S1, S2, S7 are regenerative states while the states S3, S4, S5, S6, S8, S9, S10, S11 are non- regenerative states

\section{TRANSITION PROBABILITIES AND MEAN SOJOURN TIMES}

Simple probabilistic considerations yield the following expressions for the non-zero elements.

$p_{i j}=Q_{i j}(\infty)=\int_{0}^{\infty} q_{i j}(t) d t$

$p_{01}=\frac{a x_{1}}{a x_{1}+b x_{2}}, \quad p_{07}=\frac{b x_{2}}{a x_{1}+b x_{2}}, \quad p_{10}=h^{*}\left(a x_{1}+\right.$ $\left.b x_{2}+\mu\right)$

$p_{12}=\frac{\mu}{a x_{1}+b x_{2}+\mu}\left\{1-h^{*}\left(a x_{1}+b x_{2}+\mu\right)\right\}, \quad p_{13}=$

$\frac{a x_{1}}{a x_{1}+b x_{2}+\mu}\left\{1-h^{*}\left(a x_{1}+b x_{2}+\mu\right)\right\}$

$p_{18}=\frac{b x_{2}}{a x_{1}+b x_{2}+\mu}\left\{1-h^{*}\left(a x_{1}+b x_{2}+\mu\right)\right\}, \quad p_{21}=$ $s^{*}\left(a x_{1}+b x_{2}\right)$

$p_{26}=\frac{a x_{1}}{a x_{1}+b x_{2}}\left\{1-s^{*}\left(a x_{1}+b x_{2}\right)\right\}, \quad p_{2,10}=$ $\frac{b x_{2}}{a x_{1}+b x_{2}}\left\{1-s^{*}\left(a x_{1}+b x_{2}\right)\right\}$

$$
\begin{aligned}
& p_{70}=u^{*}(0), \quad p_{11.3}=\frac{a x_{1}}{a x_{1}+b x_{2}+\mu}\{1- \\
& \left.h^{*}\left(a x_{1}+b x_{2}+\mu\right)\right\} h^{*}(0) \\
& p_{11.3(45)^{n}}=\frac{a x_{1}}{a x_{1}+b x_{2}+\mu}\left\{1-h^{*}\left(a x_{1}+b x_{2}+\mu\right)\right\} s^{*}(0) \\
& p_{17.8}=\frac{b x_{2}}{a x_{1}+b x_{2}+\mu}\left\{1-h^{*}\left(a x_{1}+b x_{2}+\mu\right)\right\} h^{*}(0) \\
& p_{17.8(9,11)^{n}}=\frac{b x_{2}}{a x_{1}+b x_{2}+\mu}\left\{1-h^{*}\left(a x_{1}+b x_{2}+\mu\right)\right\} s^{*}(0) \\
& p_{21.65}=\frac{a x_{1}}{a x_{1}+b x_{2}}\left\{1-s^{*}\left(a x_{1}+b x_{2}\right)\right\} s^{*}(0) h^{*}(0), \\
& p_{27.10,11}=\frac{b x_{2}}{a x_{1}+b x_{2}}\left\{1-s^{*}\left(a x_{1}+b x_{2}\right)\right\} s^{*}(0) h^{*}(0) \\
& p_{27.10(11,9)^{n}}=\frac{b x_{2}}{a x_{1}+b x_{2}}\left\{1-h^{*}\left(a x_{1}+b x_{2}\right)\right\}\left\{1-h^{*}(\mu)\right\} \\
& p_{45}=p_{65}=p_{9,11}=p_{10,11}=s^{*}(0), \quad p_{31}=p_{51}=p_{87}= \\
& p_{11,7}=h^{*}(\mu) \quad \\
& p_{34}=p_{54}=p_{89}=p_{11,9}=1-h^{*}(\mu)
\end{aligned}
$$

For a perfect distribution

$$
\begin{gathered}
\begin{array}{c}
p_{01}+p_{07}=p_{10}+p_{12}+p_{13}+p_{18}=p_{21}+p_{26}+p_{2,10} \\
=p_{31}+p_{34}=p_{51}+p_{54}
\end{array} \\
\begin{array}{c}
=p_{87}+p_{89}=p_{11,7}+p_{11,9}=p_{45}=p_{65}=p_{9,11}=p_{10,11} \\
=p_{10}+p_{12}+p_{11.3}+p_{17.8}
\end{array} \\
=p_{10}+p_{12}+p_{11.3(45)^{n}+p_{17.8(9,11)^{n}}} \\
=p_{21}+p_{21.65}+p_{27.10(11,9)^{n}} \\
=p_{21}+p_{21.65}+p_{27.10,11}=1
\end{gathered}
$$

\subsection{Mean Sojourn Times (MST)}

$\mu_{0}=m_{01}+m_{07}$,

$\mu_{1}=m_{10}+m_{12}+m_{13}+m_{18}$,

$\mu_{2}=m_{21}+m_{26}+m_{2,10}$

$\mu_{3}=m_{31}+m_{34}, \mu_{4}=m_{45}$,

$\mu_{5}=m_{51}+m_{54}, \quad \mu_{6}=m_{65}, \quad \mu_{7}=m_{70}$

$\mu_{8}=m_{87}+m_{89}, \mu_{9}=m_{9,11}$

$\mu_{10}=m_{10,11}, \quad \mu_{11}=m_{11,7}+m_{11,9}$

$\mu_{1}^{\prime}=m_{10}+m_{12}+m_{11.3}$

$$
+m_{17.8}+m_{11.3(45)^{n}}++m_{11.7(9,11)^{n}}
$$

$\mu_{2}^{\prime}=m_{21}+m_{26}+m_{21.65}+\quad m_{27.10,11}+m_{27.10(11,9)^{n}}$

\subsection{Reliability and Mean Time to System Failure (MTSF)}

The expressions for $\phi i(t)$ in terms of Qij( $(t)$ are as follows:

$$
\begin{aligned}
& \phi 0(\mathrm{t})=\mathrm{Q} 01(\mathrm{t}) \stackrel{\&}{\& 1(\mathrm{t})+\mathrm{Q} 07(\mathrm{t})} \\
& \phi 1(\mathrm{t})=\mathrm{Q} 10(\mathrm{t}) \stackrel{\text { \& }}{\&}{ }_{\phi 0(\mathrm{t})+\mathrm{Q} 12(\mathrm{t})}^{\&} \phi 2(\mathrm{t})+\mathrm{Q} 13(\mathrm{t})+\mathrm{Q} 18(\mathrm{t}) \\
& \phi 2(\mathrm{t})=\mathrm{Q} 21(\mathrm{t})
\end{aligned}
$$

Taking LST of expressions and solving for $\phi^{* *} 0(\mathrm{~s})$, 
We have

$$
\mathrm{R} *(\mathrm{~s})=\frac{1-\phi_{0}^{* *}(s)}{s}
$$

By taking Laplace inverse transform of the above result, the reliability of the system model can be obtained. The MTSF is given by

$\mathrm{MTSF}=\lim _{s \rightarrow 0} \frac{1-\phi_{0}^{* *}(s)}{s}=\frac{N_{1}}{D_{1}}$

Where $\mathrm{N} 1=(1-\mathrm{p} 21 \mathrm{p} 12) \mu 0+\mathrm{p} 01 \mu 1+\mathrm{p} 01 \mathrm{p} 12 \mu 2$

And $\quad$ D1 $=1-\mathrm{p} 21 \mathrm{p} 12-\mathrm{p} 01 \mathrm{p} 10$

\subsection{Steady State Availability}

The expressions for $\mathrm{Ai}(\mathrm{t})$ in terms of transition probabilities are given as:

$\mathrm{A} 0(\mathrm{t})=\mathrm{M} 0(\mathrm{t})+\mathrm{q} 01(\mathrm{t})(C \mathrm{~A} 1(\mathrm{t})+\mathrm{q} 07(\mathrm{t})(C \mathrm{~A} 7(\mathrm{t})$

$\mathrm{A} 1(\mathrm{t})=\mathrm{M} 1(\mathrm{t})+\mathrm{q} 10(\mathrm{t})(\mathrm{A} 0(\mathrm{t})+[\mathrm{q} 11.3(\mathrm{t})+\mathrm{q} 11.3(45) \mathrm{n}(\mathrm{t})](\odot)$ $\mathrm{A} 1(\mathrm{t})+\mathrm{q} 12(\mathrm{t}) \odot \mathrm{A} 2(\mathrm{t})+\quad[\mathrm{q} 17.8(\mathrm{t})+\mathrm{q} 17.8(9,11) \mathrm{n}(\mathrm{t})](C)$ $\mathrm{A} 7(\mathrm{t})$

$\mathrm{A} 2(\mathrm{t})=\mathrm{M} 2(\mathrm{t})+[\mathrm{q} 21(\mathrm{t})+\mathrm{q} 21.65(\mathrm{t})]$ C $\mathrm{A} 1(\mathrm{t})+[\mathrm{q} 27.10,11$ $(\mathrm{t})+\mathrm{q} 27.10(11,9) \mathrm{n}(\mathrm{t})]$ (C) A7(t)

$\mathrm{A} 7(\mathrm{t})=\mathrm{q} 70(\mathrm{t})(\mathrm{C} \mathrm{A} 0(\mathrm{t})$

Where M0 ( $\mathrm{t})=e^{-\left(a x_{1}+b x_{2}\right) t}, \quad \mathrm{M} 1(\mathrm{t})=e^{-\left(a x_{1}+b x_{2}+\mu\right) t} \overline{H(t)}$, M2 (t) $=e^{-\left(a x_{1}+b x_{2}\right) t} \overline{S(t)}$

Taking LT of expressions and solving for $A_{0}^{*}(s)$, the steady state availability is given by

$$
A_{0}(\infty)=\lim _{s \rightarrow 0} s A_{0}^{*}(s)
$$

$=\frac{N_{2}}{D_{2}}$

Where,

$N_{2}=\{1-(\mathrm{p} 11.3+\mathrm{p} 13+\mathrm{p} 12 \mathrm{p} 21+\mathrm{p} 12 \mathrm{p} 21.65)\} \mu 0+\mathrm{p} 01 \mu 1+$ p01 p12 42

And

$D_{2}=\left\{1-\left(p_{11.3}+p_{13}+p_{12} p_{21}+p_{21} p_{21.65}\right\} \mu_{0}+\right.$

$p_{01} p_{12} \mu_{1}+\left\{1-\left(p_{12} p_{21}+p_{21} p_{21.65}+p_{01} p_{10}+\right.\right.$

$\left.\left.p_{11.3}\right)\right\} \mu_{7}+p_{01} \mu_{1}^{\prime}+p_{01} p_{12} m_{27.10(11,9)^{n}}-m_{21} p_{70}$

\subsection{Busy Period of the Server Due to Hardware Repair}

Let $B_{i}^{H}(t)$ be the probability that the server is busy in repairing the unit due to hardware failure at an instant ${ }^{6} t$ ' given that the system entered state $S_{i}$ at $t=0$. The recursive relations for $B_{i}^{H}(t)$ are as follows:

$$
\begin{aligned}
& B_{0}^{H}(t)=q_{01}(t) \Subset B_{1}^{H}(t)+q_{07}(t) \subseteq B_{7}^{H}(t) \\
& B_{1}^{H}(t)=W_{1}^{H}(t)+q_{10}(t) \oplus B_{0}^{H}(t) \\
& +\left[q_{11.3}(t)+q_{11.3(45)^{n}}(t)\right] \subset B_{1}^{H}(t) \\
& +q_{12}(t) \mathbb{C} B_{2}^{H}(t) \\
& +\left[q_{17.8}(t)+q_{17.8(9,11)^{n}}(t)\right] \subset B_{7}^{H}(t) \\
& B_{2}^{H}(t)=\left[q_{21}(t)+q_{21.65}(t)\right] \oplus B_{1}^{H}(t)+\left[q_{27.10,11}(t)\right. \\
& \left.+q_{27.10(11,9)^{n}}(t)\right] \mathbb{C} B_{7}^{H}(t)
\end{aligned}
$$

$B_{7}^{H}(t)=q_{70}(t) \subseteq B_{0}^{H}(t)$

Now

$$
B_{0}^{H}(\infty)=\lim _{s \rightarrow 0} s B_{0}^{*^{H}}(S)=\lim _{s \rightarrow 0} s \frac{N_{3}}{D_{2}}
$$

Where

$W_{1}^{H}(t)=$

$e^{-\left(a x_{1}+b x_{2}+\mu\right) t} \overline{H(t)}+$

$\left[a x_{1} e^{-\left(a x_{1}+b x_{2}+\mu\right) t}\left(\mathcal{C} e^{-\mu t}(\mathrm{Cs}(\mathrm{t}) \mathbb{C} 1] \overline{H(t)}+\right.\right.$

$\left[b x_{2} e^{-\left(a x_{1}+b x_{2}+\mu\right) t} \mathbb{C} \mu e^{-\mu t} \mathrm{Cs}(\mathrm{t})(\mathrm{C} 1] \overline{H(t)}\right.$

$N_{3}=W_{1}^{* H}(0)\left(p_{01}+p_{12} p_{21}++p_{12} p_{21.65}\right)$ and D2 is already specified.

\subsection{Expected Numbers of Up-gradation Due to software}

Let $B_{i}^{S}(t)$ be the probability that the server is busy due to replacement of the software at an instant ' $t$ ' given that the system entered the regenerative state $S_{i}$ at $t=0$. We have the following recursive relations for $B_{i}^{S}(t)$ are as follows:

$B_{0}^{S}(t)=q_{01}(t) \subseteq B_{1}^{S}(t)+q_{07}(t) \subseteq B_{7}^{S}(t)$

$B_{1}^{S}(t)=q_{10}(t) \odot B_{0}^{S}(t)+\left[q_{11.3}(t)+q_{11.3(45)^{n}}(t)\right] \subset B_{1}^{S}(t)+$ $q_{12}(t) \odot B_{2}^{S}(t)+\left[q_{17.8}(t)+q_{17.8(9,11)^{n}}(t)\right] \subset B_{7}^{S}(t)$

$$
\begin{gathered}
B_{2}^{S}(t)=\left[q_{21}(t)+q_{21.65}(t)\right] \odot B_{1}^{S}(t)+\left[q_{27.10,11}(t)\right. \\
\left.+q_{27.10(11,9)^{n}}(t)\right] \odot B_{7}^{S}(t)
\end{gathered}
$$

$B_{7}^{S}(t)=q_{70}(t) \subseteq\left[1+B_{0}^{S}(t)\right]$

Now

$B_{0}^{S}(\infty)=\lim _{\mathrm{s} \rightarrow 0} \mathrm{~s} B_{0}^{*^{s}}(S)=\lim _{\mathrm{s} \rightarrow 0} \mathrm{~s} \frac{\mathrm{N}_{4}}{\mathrm{D}_{2}}$

$N_{4}=p_{01} p_{12}\left(p_{21}-p_{17.8}-p_{18}\right)+p_{07}\left(p_{12}+p_{11.3}\right)+$ $p_{12}\left(p_{21}+p_{21.65}\right)-\left(p_{07}+p_{12}\right)$ and $\mathrm{D} 2$ is already specified.

\subsection{Expected Numbers of Treatments Given to the Server}

Let $T_{i}(t)$ be the expected number of treatment given to the server in $(0, t]$ such that the system entered regenerative state $i$ at $\mathrm{t}=0$. The recursive relation for $T_{i}(t)$ are as follows:

$$
\begin{aligned}
& T_{0}(t)=Q_{01}(t) \& T_{1}(t)+Q_{07}(t) \& T_{7}(t) \\
& T_{1}(t)=Q_{10}(t) \& T_{0}(t)+Q_{11.3}(t) \& T_{1}(t)+Q_{11.3(45)^{n}}(t) \\
& \&\left[1+T_{1}(t)\right]+Q_{12}(t) \& T_{2}(t)+Q_{17.8}(t) \& T_{7}(t) \\
& +Q_{17.8(9,11)^{n}}(t) \&\left[1+T_{7}(t)\right] \\
& T_{2}(t)=\left[Q_{21}(t)+Q_{21.65}(t)\right] \&[1 \\
& \left.+T_{1}(t)\right]\left[Q_{27.10,11}(t)\right. \\
& \left.+Q_{27.10(11,9)^{n}}(t)\right] \&\left[1+T_{7}(t)\right]
\end{aligned}
$$

$T_{7}(t)=Q_{70}(t) \& T_{0}(t)$

Now

$\mathrm{T}_{0}(\infty)=\lim _{\mathrm{s} \rightarrow 0} \mathrm{~s} T_{0}^{*}(S)=\lim _{\mathrm{s} \rightarrow 0} \mathrm{~s} \frac{\mathrm{N}_{5}}{\mathrm{D}_{2}}$

$N_{5}=p_{01}\left(1-p_{10}-p_{12} p_{26}\right)$ and D2 is already specified.

\section{PROFIT ANALYSIS}

Profit $=\mathrm{K}_{0} \mathrm{~A}_{0}-\mathrm{K}_{1} \mathrm{~B}_{0}^{\mathrm{H}}-\mathrm{K}_{2} \mathrm{~B}_{0}^{\mathrm{S}}-\mathrm{K}_{3} \mathrm{~T}_{0}$

$\mathrm{K} 0=$ Revenue per unit up-time

$\mathrm{K} 1=$ Cost per unit time hardware repair

$\mathrm{K} 2=$ Cost per unit time software Up-gradation

K3=Cost per unit time Treatments Given to the Server 


\section{Particular Cases}

Let us take $h(t)=\alpha e-\alpha t, u(t)=\beta e-\beta t, s(t)=\gamma e-\gamma t$

We have

$$
\begin{aligned}
& h^{*}(s)=\frac{\alpha}{\alpha+s}, \quad u^{*}(s)=\frac{\beta}{\beta+s}, \\
& s^{*}(s)=\frac{\gamma}{\gamma+s} \\
& p_{01}=\frac{a x_{1}}{a x_{1}+b x_{2}}, \quad p_{07}=\frac{b x_{2}}{a x_{1}+b x_{2}}, \\
& p_{10}=\frac{\alpha}{\alpha+a x_{1}+b x_{2}+\mu} \quad p_{12}=\frac{\mu}{\alpha+a x_{1}+b x_{2}+\mu} \\
& p_{13}=\frac{a x_{1}}{\alpha+a x_{1}+b x_{2}+\mu}, \quad p_{18}=\frac{b x_{2}}{\alpha+a x_{1}+b x_{2}+\mu}, \quad p_{21}=\frac{\gamma}{\gamma+a x_{1}+b x_{2}}, \\
& p_{26}=\frac{a x_{1}}{\gamma+a x_{1}+b x_{2}} \\
& p_{2,10}=\frac{b x_{2}}{\gamma+a x_{1}+b x_{2}},
\end{aligned}
$$$$
p_{17.8}=\frac{b x_{2}}{\alpha+a x_{1}+b x_{2}+\mu},
$$$$
p_{17.8(9,11)^{n}}=\frac{b x_{2}}{\alpha+a x_{1}+b x_{2}+\mu},
$$$$
p_{21.65}=\frac{a x_{1}}{\gamma+a x_{1}+b x_{2}}
$$$$
p_{27.10,11}=\frac{b x_{2}}{\gamma+a x_{1}+b x_{2}}
$$$$
p_{27.10(11,9)^{n}}=\frac{\mu b x_{2}}{\left(\gamma+a x_{1}+b x_{2}\right)(\alpha+\mu)}
$$$$
p_{70}=p_{45}=p_{65}=p_{9,11}=p_{10,11}=1, \quad p_{31}=p_{51}=p_{87}=
$$$$
p_{11,7}=\frac{\alpha}{\alpha+\mu}
$$$$
p_{34}=p_{54}=p_{89}=p_{11,9}=\frac{\mu}{\alpha+\mu},
$$$$
\mu_{0}=\frac{1}{a x_{1}+b x_{2}}, \mu_{1}=\frac{1}{\alpha+a x_{1}+b x_{2}+\mu}
$$$$
\mu_{2}=\frac{1}{\gamma+a x_{1}+b x_{2}}, \quad \mu_{3}=\frac{1}{\alpha+\mu}, \quad \mu_{4}=\frac{1}{\gamma}, \quad \mu_{5}=\frac{1}{\alpha+\mu}, \quad \mu_{6}=\frac{1}{\gamma},
$$$$
\mu_{7}=\frac{1}{\beta}, \mu_{8}=\frac{1}{\alpha+\mu}
$$$$
\mu_{9}=\frac{1}{\gamma}, \mu_{10}=\frac{1}{\gamma}, \mu_{11}=\frac{1}{\alpha+\mu}
$$$$
\mu_{1}^{\prime}=
$$$$
(\mu+
$$

$2 a x 1+b x 21 \alpha+a x 1+b x 2+\mu-\alpha \alpha+a x 1+b x 2+\mu 2 a x 1+b \times 2+$ $\mu+\alpha \alpha+a \times 1+b \times 2+\mu 2-(a \times 1+b \times 2)(\alpha+\gamma) \alpha+a x 1+b \times 2+\mu \alpha \gamma$
$\operatorname{MTSF}=\frac{N_{1}}{D_{1}}, \quad$ Availability $\left(A_{0}\right)=\frac{N_{2}}{D_{2}}$

Busy Period of the Server Due to Hardware Repair $\left(B_{0}^{H}\right)=$ $\frac{\mathrm{N}_{3}}{\mathrm{D}_{2}}$

Expected Numbers of Up-gradation Due to software $\left(B_{0}^{S}\right)=$ $\frac{\mathrm{N}_{4}}{\mathrm{D}_{2}}$

Expected Numbers of Treatments Given to the Server $\left(\mathrm{T}_{0}\right)=\frac{\mathrm{N}_{5}}{\mathrm{D}_{2}}$

Where,

$N_{1}=$

$\left\{1-\frac{\mu \gamma}{\left(\alpha+a x_{1}+b x_{2}+\mu\right)\left(\gamma+a x_{1}+b x_{2}\right)}\right\} \frac{1}{a x_{1}+b x_{2}}+$ $\frac{a x_{1}}{\left(\alpha+a x_{1}+b x_{2}+\mu\right)\left(a x_{1}+b x_{2}\right)}+\frac{\mu a x_{1}}{\left(\alpha+a x_{1}+b x_{2}+\mu\right)\left(a x_{1}+b x_{2}\right)\left(\gamma+a x_{1}+b x_{2}\right)}$

$D_{1}=1-\frac{\mu \gamma}{\left(\alpha+a x_{1}+b x_{2}+\mu\right)\left(\gamma+a x_{1}+b x_{2}\right)}-\frac{\alpha a x_{1}}{\left(\alpha+a x_{1}+b x_{2}+\mu\right)\left(a x_{1}+b x_{2}\right)}$

$N_{2}=\left\{1-\left(\frac{2 a x_{1}}{\left(\alpha+a x_{1}+b x_{2}+\mu\right)}+\frac{\mu \gamma}{\left(\alpha+a x_{1}+b x_{2}+\mu\right)\left(\beta+a x_{1}+b x_{2}\right)}+\right.\right.$ $\mu a x 1 \alpha+a x 1+b x 2+\mu(\gamma+a x 1+b \times 2) 1 a x 1+b x 2+a x 1 \alpha+a x 1$ $+b \times 2+\mu(a \times 1+b \times 2)+\mu a x 1 \alpha+a \times 1+b \times 2+\mu(a \times 1+b \times 2)(\gamma+$ $a \times 1+b \times 2)$

$D_{2}=$

$\left\{1-\left(\frac{2 a x_{1}}{\left(\alpha+a x_{1}+b x_{2}+\mu\right)}+\frac{2 \mu \gamma}{\left(\alpha+a x_{1}+b x_{2}+\mu\right)\left(\gamma+a x_{1}+b x_{2}\right)}\right)\right\} \frac{1}{a x_{1}+b x_{2}}+$ $\left\{1-\left(\frac{a x_{1}}{\left(\alpha+a x_{1}+b x_{2}+\mu\right)}+\frac{\alpha a x_{1}}{\left(\alpha+a x_{1}+b x_{2}+\mu\right)\left(a x_{1}+b x_{2}\right)}+\right.\right.$ $\mu \gamma+\mu a \times 1 \alpha+a x 1+b x 2+? \gamma+a \times 1+b \times 21 \beta+a \times 1 a \times 1+b \times 2 \mu+$ $2 a \times 1+b \times 21 \alpha+a \times 1+b \times 2+\mu-\alpha \alpha+a \times 1+b \times 2+\mu 2 a \times 1+b \times 2+$ $\mu+\alpha \alpha+a x 1+b x 2+\mu 2-a x 1+b \times 2 \alpha+\gamma \alpha+a x 1+b \times 2+\mu \alpha \gamma+\mu$ $2 a \times 1 b \times 2 a \times 1+b \times 2 \alpha+a \times 1+b \times 2+\mu \alpha+\mu-\gamma \gamma+a \times 1+b \times 22$

$N_{3}=$

$\left(\frac{1}{\left(\alpha+a x_{1}+b x_{2}+\mu\right)}-\frac{\mu}{(\alpha+\gamma)(\alpha+\mu)}+\right.$

$\mu \gamma \mu-\gamma a \times 1+b \times 2+\gamma \alpha+a \times 1+b \times 2+\gamma+\mu \gamma \mu-\gamma a \times 1+b \times 2+\mu \alpha+$ $a \times 1+b \times 2+\mu a x 1 a \times 1+b \times 2+\mu \gamma+\mu a x 1 \alpha+a x 1+b \times 2+\mu \gamma+a x$ $1+b \times 2$

$N_{4}=$

$\frac{\mu a x_{1}}{\left(a x_{1}+b x_{2}\right)\left(\alpha+a x_{1}+b x_{2}+\mu\right)}\left(\frac{\gamma}{\left(a x_{1}+b x_{2}+\gamma\right)}-\right.$

$2 b \times 2 \alpha+a \times 1+b \times 2+\mu+b \times 2(\mu+a \times 1) a \times 1+b \times 2 \alpha+a \times 1+b \times 2+$ $\mu+\mu \gamma+a \times 1 \alpha+a \times 1+b \times 2+\mu \gamma+a \times 1+b \times 2-b \times 2 a \times 1+b \times 2+\mu \alpha$ $+a \times 1+b x 2+\mu$

$N_{5}=$

$\frac{a x_{1}}{\left(a x_{1}+b x_{2}\right)}\left(1-\frac{\alpha}{\left(\alpha+a x_{1}+b x_{2}+\mu\right)}-\frac{\mu a x_{1}}{\left(\alpha+a x_{1}+b x_{2}+\mu\right)\left(\gamma+a x_{1}+b x_{2}\right)}\right)$ 


\section{GRAPHICAL AND NUMERICAL PRESENTATIONS}

Table: 1 MTSF Vs Server Failure Rate

\begin{tabular}{|c|c|c|c|}
\hline & $\begin{array}{c}\alpha=2, \gamma=3, \mathrm{x}_{1}=0.01, \\
\mathrm{x}_{2}=0.001, \\
\mathrm{a}=0.6, \mathrm{~b}=0.4\end{array}$ & $\begin{array}{c}\alpha=3, \gamma=3, \mathrm{x}_{1}=0.01, \\
\mathrm{x}_{2}=0.001, \\
\mathrm{a}=0.6, \mathrm{~b}=0.4\end{array}$ & $\begin{array}{c}\alpha=2, \gamma=4, \mathrm{x}_{1}=0.01, \\
\mathrm{x}_{2}=0.001, \\
\mathrm{a}=0.6, \mathrm{~b}=0.4\end{array}$ \\
\hline$\mu$ & 2389.84 & 2425.359 & 2390.683 \\
\hline 0.1 & 2386.48 & 2423.037 & 2388.161 \\
\hline 0.2 & 2383.13 & 2420.72 & 2385.645 \\
\hline 0.4 & 2379.79 & 2418.408 & 2380.627 \\
\hline 0.5 & 2376.46 & 2416.1 & 2378.127 \\
\hline 0.6 & 2373.14 & 2413.798 & 2375.632 \\
\hline 0.7 & 2369.83 & 2411.5 & 2373.142 \\
\hline 0.8 & 2366.53 & 2409.208 & 2370.658 \\
\hline 0.9 & 2363.241 & 2406.92 & \\
\hline
\end{tabular}

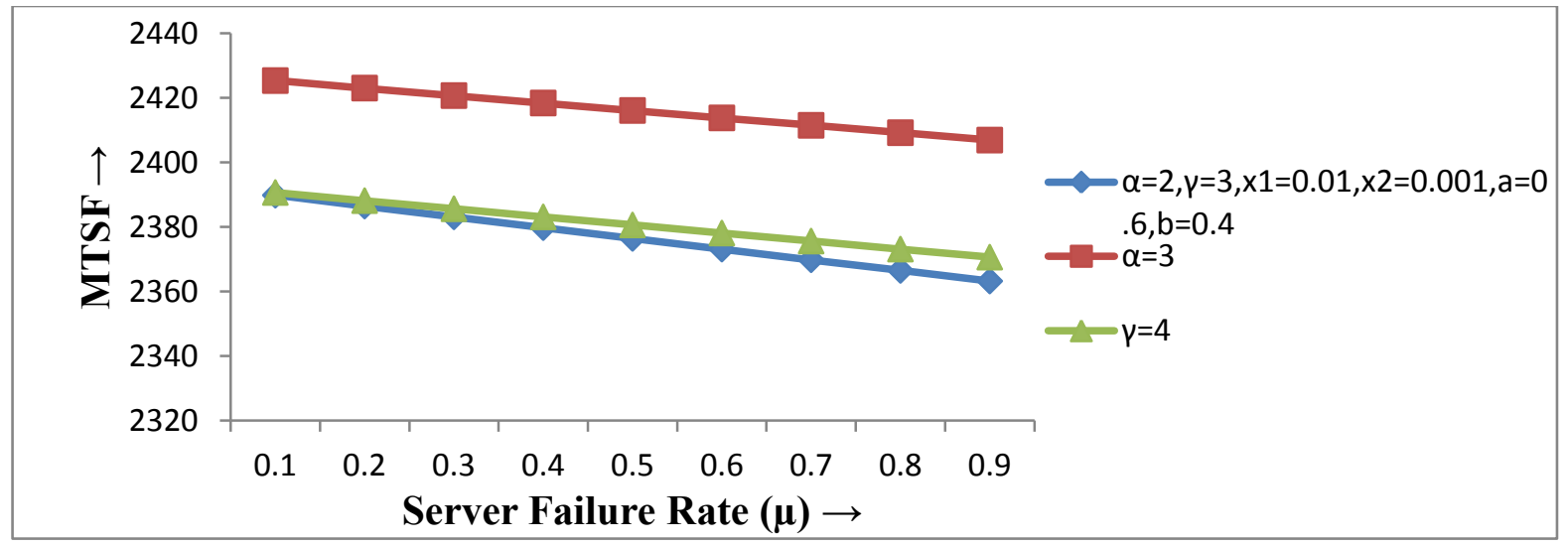

Fig. 2 MTSF Vs Server Failure Rate

Table 2 Availability Vs Server Failure Rate

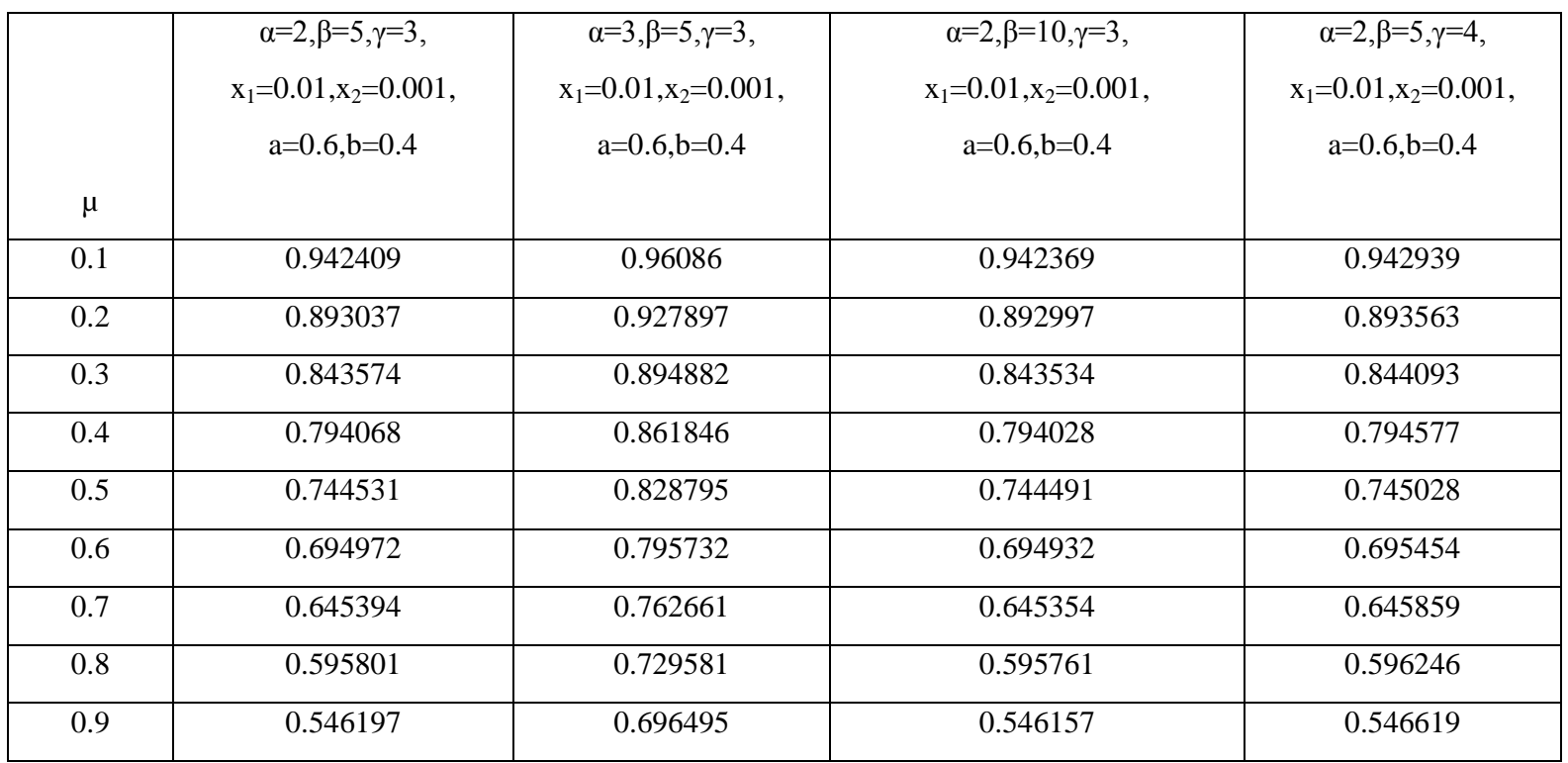




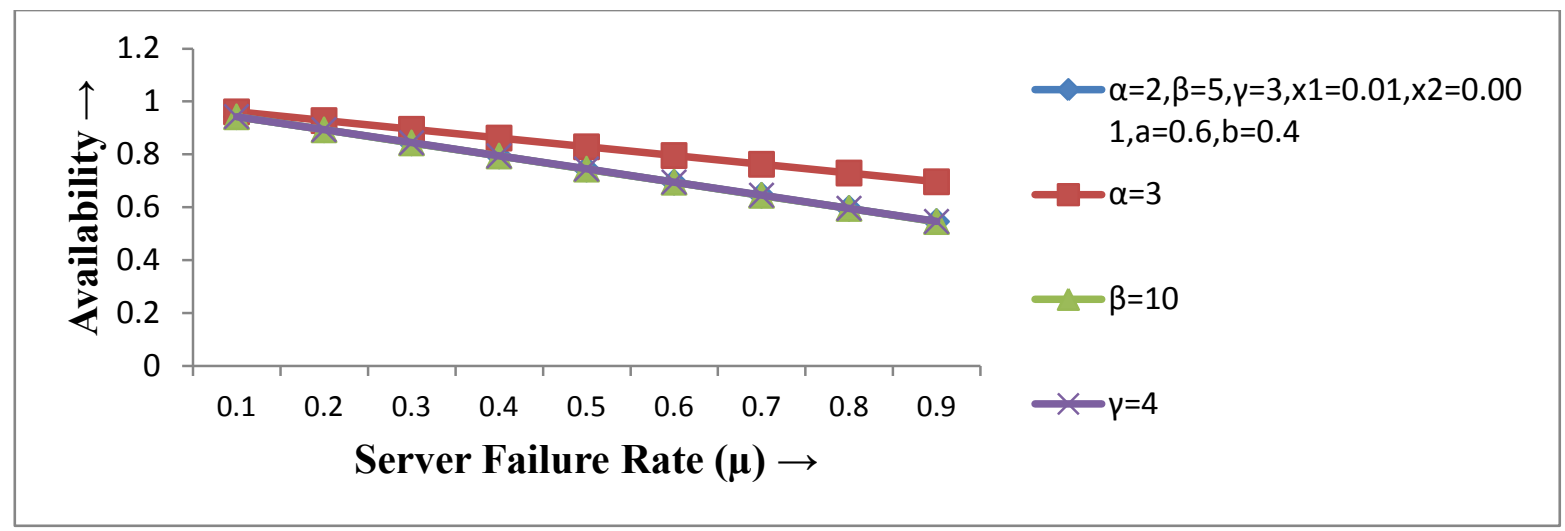

Fig. 3 Availability Vs Server Failure Rate

\begin{tabular}{|c|c|c|c|c|}
\hline \multicolumn{1}{|c|}{ Table 3 Profit Vs Server Failure Rate } \\
& $\begin{array}{c}\alpha=2, \beta=5, \gamma=3, \\
\mathrm{x}_{1}=0.01, \mathrm{x}_{2}=0.001, \\
\mathrm{a}=0.6, \mathrm{~b}=0.4\end{array}$ & $\begin{array}{c}\alpha=3, \beta=5, \gamma=3, \\
\mathrm{x}_{1}=0.01, \mathrm{x}_{2}=0.001, \\
\mathrm{a}=0.6, \mathrm{~b}=0.4\end{array}$ & $\begin{array}{c}\alpha=2, \beta=10, \gamma=3, \\
\mathrm{x}_{1}=0.01, \mathrm{x}_{2}=0.001, \\
\mathrm{a}=0.6, \mathrm{~b}=0.4\end{array}$ & $\begin{array}{c}\alpha=2, \beta=5, \gamma=4, \\
\mathrm{x}_{1}=0.01, \mathrm{x}_{2}=0.001, \\
\mathrm{a}=0.6, \mathrm{~b}=0.4\end{array}$ \\
\hline 0.1 & & & & 14170.47 \\
\hline 0.2 & 14168.17 & 14439.29 & 13467.67 & 13462.84 \\
\hline 0.3 & 13468.27 & 13977.48 & 12775.8 & 12760.75 \\
\hline 0.4 & 12776.41 & 13521.88 & 12093.84 & 12065.56 \\
\hline 0.5 & 11423.85 & 13073.85 & 11423.24 & 10699.3 \\
\hline 0.6 & 10766.21 & 12634.53 & 10765.61 & 10029.94 \\
\hline 0.7 & 10123.34 & 12205.14 & 10122.74 & 9371.045 \\
\hline 0.8 & 9497.339 & 11787.09 & 9496.737 & 8723.674 \\
\hline 0.9 & 8890.678 & 11382.01 & 8890.077 & \\
\hline
\end{tabular}

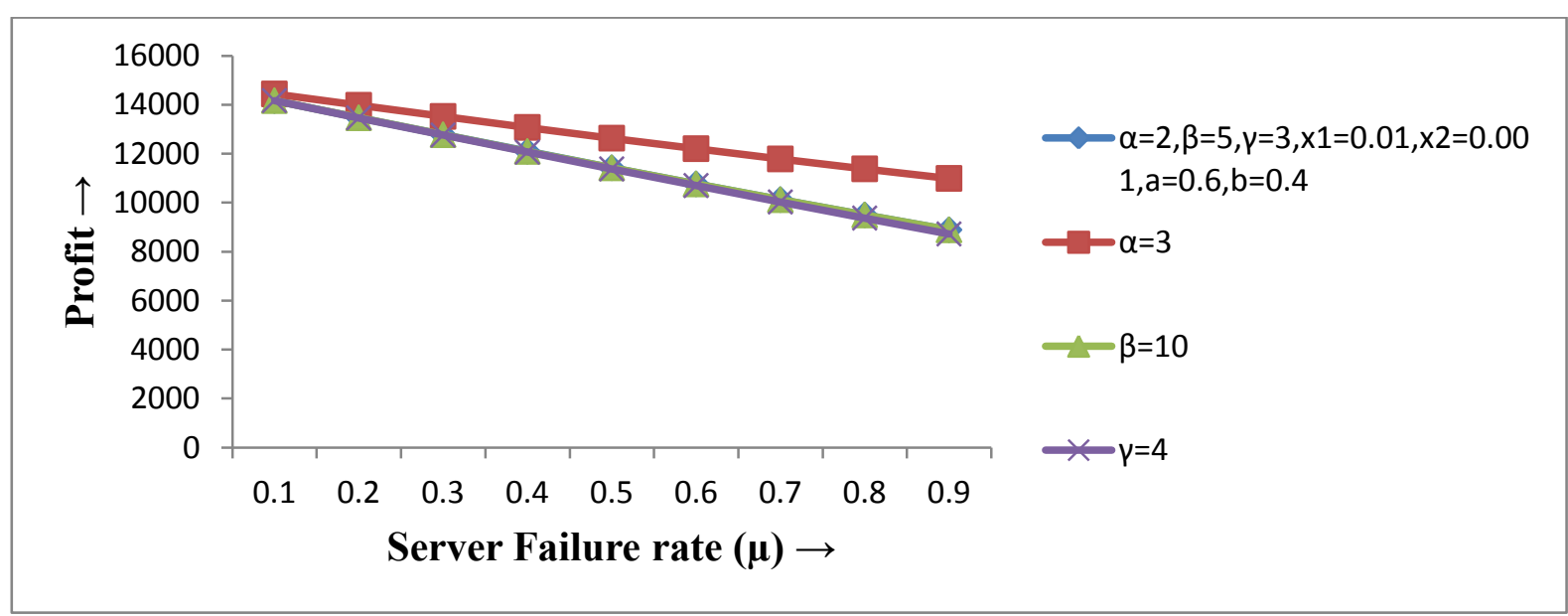

Fig. 4Profit Vs Server Failure Rate

\section{CONCLUSION}

The reliability measures of a computer system have been obtained by considering the ideas of hardware redundancy and server failure. The variation in the values of mean time to system failure, availability and profit function has been observed for arbitrary values of the parameters as shown respectively in figures $2,3 \& 4$. It is analyzed that MTSF, availability and profit incurred to the system model go on decreasing with the increase of server failure rate. However, the values of these measures keep on increasing with the increase of repair rate of the hardware and treatment rate of the server provided system has more chances of hardware failure. Hence, a computer system having more chances of hardware failure can be made reliable to use by providing cold standby redundancy to the hardware. 


\section{REFERENCES}

[1] S.C. Malik \& Anil Kumar (2010): Probabilistic Analysis of a System with Server Failure during Repair, Journal of Reliability and Statistical Studies, Vol.3(2), pp.1-10

[2] S.C. Malik \& Jyoti Anand (2010): Reliability and economic analysis of a computer system with independent hardware and software failures. Bulletin of Pure and Applied Sciences, Vol.29E(01),pp.141-153

[3] Jyoti Anand; \& S.C. Malik (2012):Probabilistic Analysis of a Computer System with Inspection and Priority for Repair Activities of H/W over Replacement of S/W. International Journal of Computer Applications, Vol.44 (1), pp. 13-21
[4] Ashish Kumar; Jyoti Anand \& S.C. Malik (2013): Stochastic Modeling of a Computer System with Priority to Up-gradation of Software over Hardware Repair Activities. International Journal of Agricultural and Statistical Sciences, Vol. 9(1), pp. 117-126

[5] S.C. Malik \& V. J. Munday (2014): Stochastic Modelling of a Computer System with Hardware Redundancy. International Journal of Computer Applications, Vol. 89(7), pp. 26-30

[6] Jyoti Nandal \& R. Rathee (2015): Stochastic Analysis of a Redundant System with Server Failure and Conditional Arrival Time. International Journal of Statistics and Reliability Engineering (IJSRE), Vol. 2(1), pp.94-102 